Article

\title{
A Method to Derive the Definition of Generalized Entropy from Generalized Exergy for Any State in Many-Particle Systems
}

\section{Pierfrancesco Palazzo}

Technip, Viale Castello della Magliana 68,00148 Rome, Italy; E-Mail: ppalazzo@technip.com or pierfrancesco.palazzo@gmail.com

Academic Editor: Marc A. Rosen

Received: 2 February 2015 / Accepted: 20 March 2015 / Published: 7 April 2015

\begin{abstract}
The literature reports the proofs that entropy is an inherent property of any system in any state and governs thermal energy, which depends on temperature and is transferred by heat interactions. A first novelty proposed in the present study is that mechanical energy, determined by pressure and transferred by work interactions, is also characterized by the entropy property. The second novelty is that a generalized definition of entropy relating to temperature, chemical potential and pressure of many-particle systems, is established to calculate the thermal, chemical and mechanical entropy contribution due to heat, mass and work interactions. The expression of generalized entropy is derived from generalized exergy, which in turn depends on temperature, chemical potential and pressure of the system, and by the entropy-exergy relationship constituting the basis of the method adopted to analyze the available energy and its transfer interactions with a reference system which may be external or constitute a subsystem. This method is underpinned by the Second Law statement enunciated in terms of existence and uniqueness of stable equilibrium for each value of energy content of the system. The equality of chemical potential and equality of pressure are assumed, in addition to equality of temperature, to be necessary conditions for stable equilibrium.
\end{abstract}

Keywords: many-particle systems; available energy; second law; stable equilibrium; non-equilibrium; generalized exergy; generalized entropy; generalized potential; generalized reservoir 


\section{Introduction}

During recent decades, Thermodynamics conceptual framework has been developed, providing the proofs that entropy is an inherent property of any system in any state, characterizing thermal energy which depends on temperature and is transferred by heat interactions. The set of all types of interactions occurring between a system $A$ and an internal subsystem or an external reference system behaving as a reservoir $R$, represent the outset for a generalization of entropy property. The reservoir $R$ is an auxiliary system, defined as adopting a duplicate of itself, experiencing stable equilibrium states only, while interacting with the system $A$ [1]. The method adopted considers entropy as a consequence of Second Law enunciated in terms of existence and uniqueness of stable equilibrium for each value of energy content of a system. Generalized entropy is obtained from generalized exergy on the basis of the definition of thermodynamic entropy, derived from energy and available energy, as reported by Gyftopoulos and Beretta [1]. Necessary conditions of equal temperature, equal chemical potential and equal pressure are the consequence of stable equilibrium within the composite system-reservoir $A R$ [1]. Instead, the canonical definition of entropy is underpinned by equal temperature only since it is based on the constant temperature $T_{R}$ as the unique property of the reservoir [1-3]. The aim is here to generalize the definition of exergy property, and consequently the definition of thermodynamic entropy, considering the equality of chemical potential $\mu=\mu_{R}$ and the equality of pressure $P=P_{R}$, as conditions of chemical stable equilibrium and mechanical stable equilibrium respectively, in addition to the thermal stable equilibrium, which imply a definition of generalized entropy suitable to characterize mass, heat and work interactions.

\section{Assumptions and Method}

The analysis focuses on many-particle macroscopic open systems according to the assumptions, definitions, theorems and terminology adopted by Gyftopoulos and Beretta [1-3]. Few-particle systems are not accounted for in the present study. Systems can experience any state of equilibrium and non-equilibrium [1]. This research focuses on transient processes to specifically analyze interactions between system and reservoir without limiting the validity of the results achieved. However, for this very reason, properties and phenomena can be extended to steady-state, and bulk-flow open systems are currently adopted in more extended theoretical treatises and in experimental and industrial applications. The reference system can be external or can be a subsystem behaving as a reservoir characterized in the macroscopic domain. The reservoir is an open system exchanging energy, entropy, amounts of constituents and volume while experiencing stable equilibrium states only. The reservoir $R$ is in mutual stable equilibrium with a duplicate of itself, behaves ideally at a permanent stable equilibrium while interacting with the system $A$ and it is an auxiliary device considering that entropy is an inherent property of any system in any state and therefore does not depend either on the characteristics and thermodynamic states of the reservoir or on its parameters variations [1-3].

The properties associated to and characterizing a reservoir may be obtained by means of a system constituted by a mass larger than the system (and ideally infinitely large). As an alternative, a pure finite-mass substance in a fixed region of space at the triple-point state behaves at constant temperature considering that heat interactions with the system occur with no changes in volume [4]. Nevertheless, the triple point ensures that pressure is constant provided that the reservoir is adiabatic and able to change 
its volume as work interactions with the system occur while the two systems are in mutual neutral equilibrium [4]. System $A$ is characterized by its states of equilibrium or non-equilibrium determined by the equality or non-equality of temperature, chemical potential (also referred to as potential), and pressure.

The method adopted is based on the assumption that equality of chemical potential and equality of pressure within a system (including the subsystem behaving as a reservoir) constitute necessary conditions of stable equilibrium, in addition to the equality of temperature. These additional conditions will therefore be accounted for to define the generalized entropy which is derived by means of the following expression [1-3]:

$$
S_{1}-S_{0}=\frac{1}{C_{R}}\left[\left(E_{1}-E_{0}\right)-\left(\Omega_{1}^{R}-\Omega_{0}^{R}\right)\right]
$$

where $C_{R}$ is a constant property of the reservoir constituting an auxiliary system [5] as a matter of fact that entropy is an inherent property to any system in any state [1-3] and does not depend on the reservoir. The term $\left(\Omega_{1}^{R}-\Omega_{0}^{R}\right)$ is the difference of generalized available energy $\Omega^{R}$ at states 1 and 0 with respect to the reservoir $R$. The generalized available energy can be correlated to exergy property if a generalized reservoir is assumed at permanent stable equilibrium characterized by any combination of constant temperature $T_{R}$, constant chemical potential $\mu_{R}$ and constant pressure $P_{R}$ which may not correspond to the conditions of the environment [1]:

$$
S_{1}-S_{0}=\frac{1}{C_{R}}\left[\left(E_{1}-E_{0}\right)-\left(E X_{1}^{R}-E X_{0}^{R}\right)\right]
$$

so that entropy can be considered correlated to the equality of temperature, equality of potential and equality of pressure between a system and a reservoir consistent with the above mentioned definition. This definition of entropy property assumes that stable equilibrium is the reference state between system and reservoir; however, the state of system itself can be equilibrium or non-equilibrium [1-3]. Indeed, both energy and generalized available energy, or exergy, are properties valid in the domain of any state, equilibrium or non-equilibrium, of any system, large and small even consisting of one single particle. This also implies that the above Equations (1a) and (1b) account for both reversible and irreversible processes. In particular, by virtue of the entropy-exergy relationship: entropy contributions are correlated to exergy losses due to reversible transfer to the reservoir; and irreversible entropy generation, correlated to exergy destruction is caused by irreversible conversions along non-equilibrium processes.

\section{Thermal Entropy Related to Thermal Stable Equilibrium}

Generalized available energy $\Omega^{R}$ is an additive property because it is defined with respect to an external reference system or to an internal part behaving as a reservoir and is derived from the generalized adiabatic availability which, instead, is not additive [1-3]. The definition of exergy property is a consequence of generalized available energy and is associated with heat and work interactions occurring until the composite system-reservoir reaches the stable equilibrium. In particular, the (thermal) exergy formulated as the maximum net useful work $W_{10}^{A R \rightarrow}$, withdrawn from the composite $A R$ by means of a weight process resulting from the difference of generalized available energy between the variable temperature $T$ of system $A$ and the temperature $T_{R}$ of the reservoir $R$, is the following [1]: 


$$
E X^{T}=W_{10}^{A R \rightarrow}=\Omega_{1}^{R}-\Omega_{0}^{R}=\left(U_{1}-U_{0}\right)-T_{R} \cdot\left(S_{1}^{T}-S_{0}^{T}\right)+P_{R} \cdot\left(V_{1}-V_{0}\right)
$$

The term $P_{R} \cdot\left(V_{1}-V_{0}\right)$ expresses the amount of work released to the reservoir at constant pressure $P_{R}$ differing from the (variable) pressure $P$ of the system which therefore is not in mutual stable equilibrium with the reservoir even though the composite system-reservoir $A R$ is in (thermal) stable equilibrium state due to the equality of temperatures $T=T_{R}$.

According to the procedure here adopted, the (thermal) entropy is derived from the difference between energy $E$ and generalized available energy $\Omega^{R}$ which are both measurable properties whereas entropy is not $[1-3]$ :

$$
\left(S_{1}-S_{0}\right)^{T}=\frac{1}{T_{R}}\left[\left(E_{1}-E_{0}\right)-\left(\Omega_{1}^{R}-\Omega_{0}^{R}\right)\right]^{T}
$$

and considering that generalized available energy and (thermal) exergy properties are correlated:

$$
\left(S_{1}-S_{0}\right)^{T}=\frac{1}{T_{R}}\left[\left(E_{1}-E_{0}\right)-\left(E X_{1}^{R}-E X_{0}^{R}\right)\right]^{T}=\frac{\Delta E_{R}^{T}}{T_{R}}
$$

This formulation of thermal entropy, as a state property intrinsic to inter-particle kinetic energy, depends on temperature, chemical potential and pressure at each state, however its definition is related only to reservoir's thermal potential, namely temperature $T_{R}$, which remains constant while interacting with the system.

The physical meaning of thermal entropy resulting from this expression consists of the amount of energy content which is not converted into work interaction and therefore constitutes the "disordered" energy distributed among all particles constituting the system. In different terms, thermal entropy represents the degree of distribution of a system's inter-particle kinetic energy amongst the available internal degrees of freedom due to the distribution of temperature inside the system [6]. Thermal entropy is therefore correlated to the amount of non-available (thermal) energy, that is exergy loss, which can only be transferred to the thermal reservoir at $T_{R}$ by means of heat interaction. If the canonical definition of entropy conceived by Clausius is considered, the temperature $T$ of the system represents the integrating factor [7] of the ratio corresponding to, in differential terms: $d S^{T}=\frac{\delta Q}{T}=\frac{d E_{R}^{T}}{T_{R}}$ which is a state and additive property while $\delta Q$ is not.

It should be noted that the above Equations (3a) and (3b) of thermal entropy do not include pressure $P_{R}$ of the mechanical reservoir which instead appears in the definition of thermal exergy $E X^{T}=W_{10}^{A R \rightarrow}=\Omega_{1}^{R}-\Omega_{0}^{R}$ in Equation (2). Moreover, the equality of temperature is a necessary condition of (thermal) stable equilibrium derived through the Highest-Entropy Principle [8], nevertheless it is not the unique condition. In fact, even though two interacting systems are in thermal stable equilibrium due to equal temperatures, these systems may experience (constraints removed) states of non-equilibrium due to a non-null difference of chemical potential and or pressure. Therefore, the equality of temperature, equality of chemical potential and equality of pressure, between system and reservoir, should constitute the set of necessary conditions ensuring the mutual stable equilibrium among all subsystems of any whole system. The proof that equal chemical potential and equal pressure are necessary conditions for 
stable equilibrium [1], is founded on the Highest-Entropy Principle in which the entropy property is defined with respect to temperature only. Hence, since the definition of thermal entropy does not account for equality of chemical potential and equality of pressure, and neither does the Highest-Entropy Principle account for these additional conditions, entropy should therefore require a generalization extended to the contributions of chemical entropy and mechanical entropy evaluated with respect to equality of chemical potential and equality of pressure within the composite system-reservoir. The two above inconsistencies will be resolved by generalizing exergy and entropy proving that chemical potential and pressure affect exergy property and consequently entropy definition.

\section{Chemical Entropy Related to Chemical Stable Equilibrium}

The potential generated by interactions among particles, atoms and molecules of a many-particle system constitutes the chemical energy (or partial molar free energy) that is defined as a form of "disordered energy", as the thermal energy is [9]. In analogy with the procedure provided for thermal entropy, a definition of chemical exergy will first be stated in terms of maximum work of reversible (internally and externally) chemical reactions, as expressed by the Gibbs function so that $W_{R E V}^{N E T}=-\Delta G$. The Van't Hoff equilibrium open system at constant temperature and constant pressure is a device suitable for reproducing a typical chemical reaction $n_{A} A+n_{B} B \leftrightarrow n_{C} C+n_{D} D$ where A, B are reactants and C, D are products [9]. In the Van't Hoff device, reactants and or products have to be constituted by the reference substances or constituents contained in the reservoir (commonly represented by the environment). This device is adopted to calculate maximum net useful work taking into account the internal chemical reactions and physical operations required to bring those reference substances to the conditions they assume in the reservoir. The Gibbs relation, obtained from mass and internal energy balance, as a consequence of the Stable-Equilibrium-State Principle [1], is the following:

$$
\begin{gathered}
d U\left(S, n_{i}, V\right)=T \cdot d S+\sum_{i} \mu_{i} d n_{i}-P \cdot d V \\
=\left(\frac{\partial U}{\partial S}\right)_{V, n} d S+\sum_{i}\left(\frac{\partial U}{\partial n_{i}}\right)_{V, S} d n_{i}-\left(\frac{\partial U}{\partial V}\right)_{S, n} d V
\end{gathered}
$$

where the term $\mu_{i}=\left(\frac{\partial U}{\partial n_{i}}\right)_{V, S, n}$ represents the chemical potential of the $i-t h$ constituent (also referred to as "partial molar Gibbs energy").

On the basis of the definitions provided by Kotas [9], chemical exergy can be evaluated as the maximum net useful work obtainable from a constituent when it is transferred from the system to the reservoir by means of reversible processes involving mass interaction with the reservoir. Indeed, such a chemical reservoir can be characterized according to the definition proposed by Gyftopoulos and Beretta [1] as a "reservoir with variable amounts of constituents" behaving at permanent stable equilibrium while exchanging mass of constituents with the system. The expression of chemical exergy of a constituent undergoing physical operations and or chemical reactions, or a combination of the two, constituting a process from initial state 0 to final state 1 , corresponding to the maximum net useful work withdrawn from the system interacting with the chemical reservoir, is expressed by the following equation [9]: 


$$
E X^{C}=\left(W_{10}^{A R \rightarrow}\right)^{M A X}=\bar{R} T_{R} \ln \frac{P_{1}}{P_{0}}
$$

where $P_{0}$ is the partial pressure of the constituent at the initial state of the process and $P_{1}$ is the partial pressure of the same constituent at final the state of the process within the system $A$. The superscript "C" stands for "Chemical reservoir" since the composite system-reservoir undergoes an interaction that can be defined as "mass interaction". Mass interactions are characteristic of chemical energy transfer and are moved by the difference of chemical potential between the system and the chemical (isopotential) reservoir. In the general case of a mixture of $n$ constituents, chemical exergy is also defined by Moran and Sciubba as follows [10]:

$$
E X^{C}=\sum_{i}^{n}\left(W_{10}^{A R \rightarrow}\right)_{i}^{M A X}=\bar{R} T_{R} \sum_{i}^{n} x_{i, 1} \ln \frac{x_{i, 1}}{x_{i, 0}}
$$

where $x_{i}$ represents the molar fraction of the $i-t h$ constituent. An alternative expression for mixtures of $n$ constituents is $E X^{C}=\sum_{i}^{n} n_{i}\left(\mu_{i, 1}-\mu_{i, 0}\right)$ where $\mu_{i}$ represents the chemical potential of the $i-t h$ constituent [10]. Equality of chemical potentials is accounted for as an additional necessary condition for mutual stable equilibrium between the system and the reservoir, in addition to equality of temperature [1]. This implies a definition of chemical entropy derived from the chemical exergy and chemical energy according to the methodology previously adopted. To do so, if the concept of generalized available energy is now reconsidered, the formulation of chemical exergy should be translated in the expression $E X^{C}=\left(W_{10}^{A R \rightarrow}\right)^{M A X}=\left(\Omega_{1}^{R}-\Omega_{0}^{R}\right)^{C}$. Since energy and generalized available energy are additive properties, then chemical entropy is an additive property as well. Chemical entropy may be derived from chemical potential and generalized available energy which depends on mass interaction:

$$
\left(S_{1}-S_{0}\right)^{C}=\frac{1}{\mu_{R}}\left[\left(E_{1}-E_{0}\right)-\left(\Omega_{1}^{R}-\Omega_{0}^{R}\right)\right]^{C}
$$

and again considering the correlation assumed between generalized available energy and chemical exergy:

$$
\left(S_{1}-S_{0}\right)^{C}=\frac{1}{\mu_{R}}\left[\left(E_{1}-E_{0}\right)-\left(E X_{1}^{R}-E X_{0}^{R}\right)\right]^{C}=\frac{\Delta E_{R}^{C}}{\mu_{R}}
$$

expressing the chemical entropy derived from chemical energy and chemical exergy and based on the equality of potential that constitutes a necessary condition of stable equilibrium between the system and the chemical reservoir. This formulation of chemical entropy, as a state property intrinsic to inter-particle potential energy, depends on temperature, chemical potential and pressure at each state, however its definition is related only to the reservoir's chemical potential $\mu_{R}$ which remains constant while interacting with the system.

The physical meaning of chemical entropy can be associated with the amount of disordered energy released to the reservoir that can no longer be converted into useful work. In other terms, chemical entropy represents the degree of distribution of a system's chemical potential amongst the available internal degrees of freedom due to the distribution of concentration or density of the mass inside the system. This also implies that stable equilibrium should be regarded as the result of the contributions by 
both thermal equilibrium and chemical equilibrium as a result of system's internal phenomena. Equations (7a) and (7b) depend on potential of the reservoir $\mu_{R}$ formally analogous to the thermal entropy in Equations (3a) and (3b) where the term $1 / \mu_{R}$ corresponds to the term $1 / T_{R}$ both representing integrating factors which make thermal entropy and chemical entropy state properties [10]. Canonical equations of system processes refer to the variation in entropy determined by thermal energy transfers and heat interactions associated with any physical operation or chemical reaction, or a combination of the two, within the composite system-reservoir. Instead, chemical entropy is an inherent property of systems related to the distribution of inter-atomic and inter-molecular potential energy and represents the intrinsic and additional chemical contribution to the entropy balance due to mass interactions within systems undergoing physical-chemical processes during which inter-particle potentials change. In infinitesimal terms, $d S^{C}=\frac{\delta M}{\mu}=\frac{d E_{R}^{C}}{\mu_{R}}$ where and $M$ stands for mass or moli interaction, $\mu$ is the chemical potential of the system, $\mu_{R}$ is the chemical potential of the reservoir both representing integrating factors [7]. Chemical entropy is the property which can be addressed in the HighestChemical-Entropy Principle to prove that chemical stable equilibrium is a sufficient condition for chemical potential equality between system and chemical reservoir, given the equality of temperature and pressure of the composite system-reservoir.

\section{Mechanical Entropy Related to Mechanical Stable Equilibrium}

The weight process constitutes a device adopted to measure the maximum net useful work extracted from a system $A$ releasing a corresponding minimum non-useful heat to a (thermal) reservoir $R^{T}$ according to the definition of generalized available energy and thermal exergy $[1,11]$ here adopted. The inverse and reversible process requires the weight process to be the minimum net useful work released to $A$ extracting a corresponding maximum non-useful heat from $R^{T}$. Nevertheless, the weight process can also be regarded as an interaction suitable for calculating the minimum non-useful work $\left(W_{10}^{A R \rightarrow}\right)^{M I N}$, released by the system $A$ to a mechanical reservoir $R^{M}$ at constant pressure $P_{R}$, correlated to the maximum net useful heat $\left(Q_{10}^{A R \rightarrow}\right)^{M A X}$ which, in this symmetric process, can be referred to as mechanical exergy $E X^{M}$ [11]. In this case, the weight process occurs through the interaction of the system $A$ with the mechanical reservoir $R^{M}$ until the difference in pressure between system and reservoir is null. Figure 1 depicts the area TX-0-P0-PX representing this amount of non-useful work interaction released to $R^{M}$. The mechanical reservoir may be characterized by the same properties as a thermal reservoir since no specific mention of "thermodynamic potential" is made in the definition reported in the literature. Due to the fact that non-useful work is released interacting with the mechanical reservoir, it may be inferred that work, such as heat, is not useful since it constitutes the component of generalized available energy of the composite system-reservoir which is not convertible into useful heat $\left(Q_{10}^{A R \rightarrow}\right)^{M A X}$.

Minimum non-useful work can be calculated once the system has reached thermal equilibrium due to the equality of temperature of the composite system-reservoir. In fact, work interaction along the isothermal process, may not be considered useful because it must be entirely converted into useful 
heat by means of a Joule cycle releasing non-useful work to the mechanical reservoir at a lower and constant pressure. Thus, it is unavailable, so that the maximum net useful heat and the minimum non-useful work is attained. This auxiliary Joule cycle operates between different constant pressures and between equal constant temperatures by means of a two-phase operating fluid. Figure 1 describes the amount of work interaction that is converted into useful heat releasing non-useful work to the reservoir which is unable to transfer this work back to the system because of the Lowest-Energy Principle [1,7] while entropy remains constant due to the assumed reversibility of processes. If the inverse isothermal process is analyzed, work interaction from the reservoir to the system cannot occur as a spontaneous process by virtue of the Lowest-Energy Principle and needs to be forced by means of a direct ideal cycle converting heat into work. At constant lower pressure, the Joule cycle is able to accomplish this conversion between $P$ and $P_{R}$ as shown in Figure 1 .

If the whole process is located in the area on the right-hand side of the diagram with respect to the reservoir's thermodynamic coordinates, the Joule cycle is replaced by a Carnot cycle. In fact the process reaches the isobaric curve before the isothermal curve, as in the previous case.

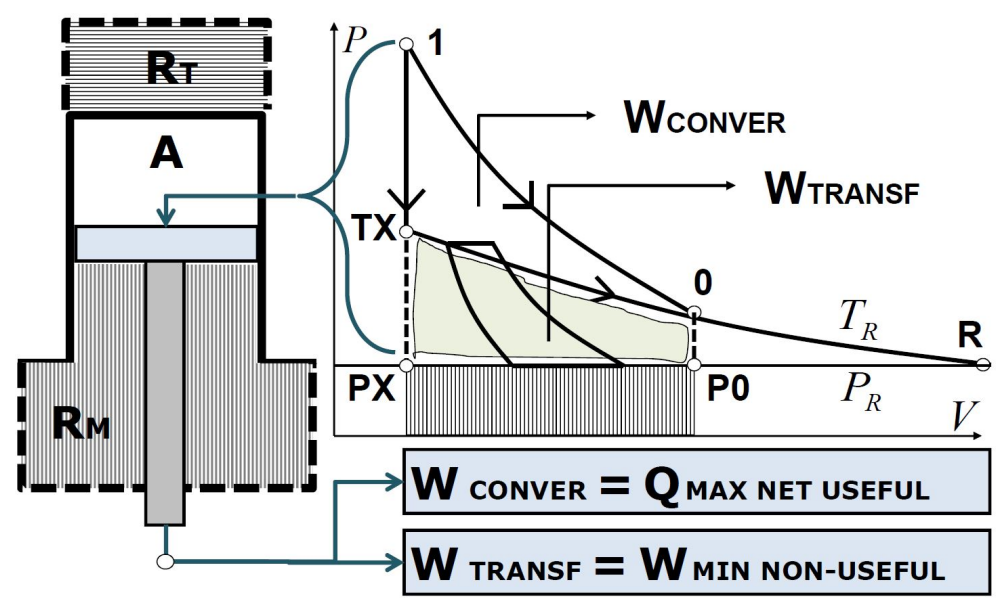

Figure 1. System interacting with thermal and mechanical reservoirs.

To summarize, the mechanical exergy property accounts for the maximum net useful heat $\left(Q_{10}^{A R \rightarrow}\right)^{M A X}$ extracted from the system $A$ releasing the minimum non-useful work to the mechanical reservoir. The system $A$ interacts with a mechanical reservoir behaving as an isobaric reservoir $R^{M}$. Then, with the symbol $E X^{M}$ adopting the superscript "M" standing for "Mechanical":

$$
E X^{M}=\left(Q_{10}^{A R \rightarrow}\right)^{M A X}=\left(\Omega_{1}^{R}-\Omega_{0}^{R}\right)^{M}
$$

This relation expresses the amount of generalized available (mechanical) energy of the system $A$ converted into heat interaction $\left(Q_{10}^{A R \rightarrow}\right)^{M A X}$ at a higher temperature with respect to the thermal reservoir $R^{T}$ at constant temperature $T_{R}$. On the other side, $\left(Q_{10}^{A R \rightarrow}\right)^{M A X}$ results from the minimum amount of work interaction $\left(W_{10}^{A R \rightarrow}\right)^{M I N}$ released to the mechanical reservoir along the isothermal process in which the heat interaction is withdrawn from the thermal reservoir to be converted into $\left(Q_{10}^{A R \rightarrow}\right)^{M A X}$. 
The minimum amount of non-useful work interaction, corresponding to the maximum net useful heat withdrawn, at constant temperature $T_{R}$, from the thermal reservoir and converted into useful heat at higher temperature, is expressed as follows:

$$
\left[\left(E_{1}-E_{0}\right)-\left(E X_{1}^{R}-E X_{0}^{R}\right)\right]^{M}=\left(W_{10}^{A R \rightarrow}\right)^{M I N}=\bar{R} T_{R}\left(\ln V_{1}-\ln V_{0}\right)=P_{R} V_{R}\left(\ln V_{1}-\ln V_{0}\right)
$$

The term $\bar{R} T_{R}\left(\ln V_{1}-\ln V_{0}\right)=P_{R} V_{R}\left(\ln V_{1}-\ln V_{0}\right)$ equals the (theoretically minimum) amount of work released to the reservoir and is equal to the low temperature heat, withdrawn from the thermal reservoir, converted into high temperature heat $\left(Q_{10}^{A R \rightarrow}\right)^{M A X}$. Moreover, because $E X^{M}=\left(Q_{10}^{A R \rightarrow}\right)^{M A X}$ then the work released along the isothermal process has to be converted into heat by means of the auxiliary Joule inverse cycle, operating between two isobaric processes, which converts the non-useful work into heat released to the thermal reservoir:

$$
\left(Q_{10}^{A R \leftarrow}\right)^{M I N}=\int_{X}^{0}\left[1-\left(\frac{P_{R}}{P}\right)^{\frac{K-1}{K}}\right] \cdot d W_{\text {ISOBARIC }}^{H P}
$$

where $d W_{I S O B A R I C}^{H P}=-P \cdot d V$ is equal to $d W_{\text {ISOTHERMAL }}^{R E S E V O I R}$, the state equation is $P=\frac{\bar{R} T_{R}}{V}$, and considering that $\left(\frac{P_{R}}{P}\right)^{\frac{K-1}{K}}=\frac{T_{R}}{T}$ and $T=T_{R}$ since the auxiliary Joule inverse cycle operates between isobaric processes at the same reservoir temperature $T_{R}$ then $Q=-\int_{X}^{0} P_{R} V_{R} \cdot \frac{d V}{V}=P_{R} V_{R} \cdot\left(\ln V_{X}-\ln V_{0}\right)$. This result proves that the minimum non-useful work is equal to minimum non-useful heat and therefore, with the terminology here adopted, the thermal entropy withdrawn from the thermal reservoir is equal to the mechanical entropy released to the mechanical reservoir so that thermal entropy is converted into mechanical entropy along the isothermal process at constant temperature $T_{R}$ while being released to the reservoir.

The definition of mechanical exergy formulated by Equation (8) is the basis for deriving the expression on mechanical entropy using the same procedure adopted for thermal entropy and chemical entropy:

$$
\left(S_{1}-S_{0}\right)^{M}=\frac{\bar{R}}{P_{R} V_{R}}\left[\left(E_{1}-E_{0}\right)-\left(E X_{1}^{R}-E X_{0}^{R}\right)\right]^{M}=\frac{\Delta E_{R}^{M}}{P_{R} V_{R}}
$$

This formulation of mechanical entropy, as a state property intrinsic to both inter-particle kinetic energy and inter-particle potential energy, depends on temperature, chemical potential and pressure at each state. Its definition is related only to the reservoir's mechanical potential $P_{R} V_{R}$ which remains constant while interacting with the system.

The above expression is founded on the Second Law relating to the existence and uniqueness of a stable equilibrium state in a composite system-reservoir $A R$. The formulation of mechanical entropy can be demonstrated considering that the proof provided by Gyftopoulos and Beretta [1] does not impose any restriction with respect to the form of energy and generalized available energy constituting 
the expression of entropy. Therefore, the same procedure can be considered still valid regardless of the physical nature of the properties involved. Moreover, by using the Highest-Mechanical-Entropy Principle, the equality of pressure can be proved to be an additional necessary condition of mutual stable equilibrium between system and reservoir that needs to comply with the equality of temperature and potential to ensure the equilibrium status of the composite system-reservoir as a whole.

The term $\bar{R} T_{R}\left(\ln V_{1}-\ln V_{0}\right)=P_{R} V_{R}\left(\ln V_{1}-\ln V_{0}\right)$ of Equation (9) substituted in the Equation (10), implies the expression of the mechanical entropy:

$$
\left(S_{1}-S_{0}\right)^{M}=\bar{R}\left(\ln V_{1}-\ln V_{0}\right)
$$

The physical meaning of mechanical entropy property is related to the distribution of particle positions within the available space of the system measured by the specific volume which coincides with the total volume in a condition of mutual stable equilibrium between system and mechanical reservoir.

An adiabatic reversible process is also defined as isoentropic since the thermal entropy change is null as heat interactions do not occur and work interactions are the unique occurring in this process. Instead, the novelty is that the adiabatic reversible process may not be defined as isoentropic since it implies a change of mechanical entropy that is not null because it relates to work interaction which transfers the mechanical available energy to the external reference system here identified as a mechanical reservoir.

The concept of mechanical entropy derived from mechanical exergy has been formalized in this section on the basis of the thermal and mechanical aspects of entropy-exergy relationship. Nevertheless, if reference is made to the chemical and mechanical aspects of this relationship, the corresponding formulation of mechanical entropy derived from mechanical exergy can be correlated to maximum mass interaction $\left(M_{10}^{A R \rightarrow}\right)^{M A X}$ which also implies minimum work interaction $\left(W_{10}^{A R \rightarrow}\right)^{M I N}$. In both cases the macroscopic thermodynamic work $W_{10}^{A R \rightarrow}=-P \Delta V$ resulting from the microscopic work is transmitted by each single particle to the container wall through collisions which are indirect, among particles, and direct between each particle and the container wall. This is the reason why thermodynamic work is as disordered as heat, and for this very reason it is not better than heat and therefore is not as ordered as work of electric, magnetic and fluid-dynamic fields. This means that it makes sense to define mechanical entropy related to thermodynamic work formulated as $d S^{M}=\frac{\delta W}{P V}=\frac{d E_{R}^{M}}{P_{R} V_{R}}$ [11] where $P V$ and $P_{R} V_{R}$ represent integrating factors [7].

\section{Generalized Exergy Related to Generalized Potential}

The definition of exergy is characterized by the property of additivity because it is defined with respect to an external reference system or to an internal part of the system itself behaving as a reservoir. The definition of a thermo-chemical-mechanical reservoir characterized by constant temperature, chemical potential and pressure implies the property of additivity of the components that constitute the generalized exergy so that:

$$
\begin{gathered}
E X^{G}\left(T, T_{R}, \mu, \mu_{R}, P, P_{R}\right)=\left(Q^{A R \rightarrow}\right)+\left(M^{A R \rightarrow}\right)+\left(W^{A R \rightarrow}\right) \\
=E X^{T}\left(T, T_{R}\right)+E X^{C}\left(\mu, \mu_{R}\right)+E X^{M}\left(P, P_{R}\right)
\end{gathered}
$$


This generalized definition of exergy property applies to both exergy losses, released to the reservoir by reversible transfer, and exergy destruction due to irreversible processes both representing all real processes occurring in any system undergoing any process.

\section{Generalized Entropy Derived from Generalized Exergy}

The additivity of the entropy property can be proved considering the additivity of energy and generalized available energy [1]. On the basis of the additivity of the entropy property [1], the generalized entropy results from the sum of entropy components each derived from the corresponding exergy component related to the (generalized) potential constituted by temperature, chemical potential and pressure. Therefore, the generalized entropy $S^{G}$ can be expressed as:

$$
S^{G}=S^{G}\left(T, T_{R}, \mu, \mu_{R}, P, P_{R}\right)=S^{T}\left(T, T_{R}\right)+S^{C}\left(\mu, \mu_{R}\right)+S^{M}\left(P, P_{R}\right)
$$

Generalized entropy is derivable from generalized exergy if, and only if, the system is brought at stable equilibrium with a generalized reservoir from any state. This final condition implies an equality of temperature, chemical potential and pressure between system and reservoir which becomes a set of necessary conditions for the stable equilibrium state of the composite system-reservoir constituting the prerequisite for calculating generalized entropy of any state of equilibrium or non-equilibrium.

Canonical equations characterizing all processes make reference to the variation of entropy determined by thermal energy transfers and heat interactions within the system and with the external reservoir. Instead, generalized entropy is inherent in all kinds of inter-particle kinetic and potential energy and represents the overall contribution to entropy balance due to all kinetic energy and potential energy determined by interactions:

$$
d S^{G}=\frac{\delta I^{G}}{P^{G}}=\frac{d E_{R}^{G}}{P_{R}^{G}}=\frac{d\left(E^{G}-E X^{G}\right)}{P_{R}^{G}}
$$

where $P^{G}$ and $P_{R}^{G}$ represent generalized potentials constituting the integrating factors [7] of $I^{G}$ standing for generalized interaction. This generalized definition of entropy property is valid for both entropy contribution, relating to reversible external and internal processes, and entropy generation produced by irreversibilities of internal processes.

\section{Conclusions}

The method adopted in the analysis herein proposed is based on the concept of the generalized available energy of a system interacting with a reservoir leading to the definition of exergy property. This method establishes the procedure for deriving entropy from energy and generalized available energy and therefore entropy from generalized exergy property. It highlights the fact that pressure takes on the function of determining the useful heat interaction converted from available mechanical energy as temperature does with respect to useful work converted from available thermal energy. This conclusion is consistent with the assumption that, in addition to the equality of temperatures, the equality of pressures between system and (thermo-mechanical) reservoir should be considered as an additional necessary condition of mutual stable equilibrium, according to the Second Law statement. Moreover, it points out that pressure also takes on the function of determining the useful mass interaction converted from 
available mechanical energy as potential does with respect to useful work converted from available chemical energy. Equality of chemical potential between system and (chemical or mass) reservoir constitutes a further additional necessary condition of stable equilibrium. Hence, the equality of "generalized" potential represents a set of conditions necessary to derive generalized entropy from the generalized exergy of a system interacting with a generalized reservoir. These conclusions are based on, and consistent with, the fundamental Highest-Generalized-Entropy Principles underpinned by the existence and uniqueness of the thermal, chemical and mechanical stable equilibrium state for each value of energy content of the system according to the Second Law statement. Nevertheless, the proofs reported in the literature do not assume any restriction relating to the status of the system which may evolve through equilibrium and non-equilibrium states.

As far as possible future researches are concerned, the method here discussed may first undergo a more rigorous formalization of properties and their definitions as here proposed, according to the recent papers of Beretta and Zanchini [12,13]. A further purpose would be that of proving both the necessity and sufficiency of stable equilibrium, already enunciated as a theorem for many-particle systems [14], also extended to few-particle systems adopting the same Beretta and Zanchini procedure to generalize the definition of thermodynamic entropy to any system, large and small, in any state, equilibrium and non-equilibrium.

A second consequence, and a possible application of generalized entropy and its components, may be the analysis of complex and biological systems as already reported in the literature [15-21] with the aim of analyzing laws governing the self-assembling and self-organizing processes of atomic and molecular interactions at microscopic level.

Finally, a question may be posed as to whether an overarching vision of availability [22] is capable of conceiving a quantum exergy, to derive a quantum entropy property [23], as a further generalization of the entropy-exergy relationship to be included within the whole framework of the Unified Quantum Theory of Mechanics and Thermodynamics.

\section{Nomenclature}

$\begin{array}{ll}A & \text { system } \\ A R & \text { composite system-reservoir } \\ C & \text { chemical } \\ E & \text { energy, } \mathrm{J} \\ E X & \text { exergy, } \mathrm{J} \\ G & \text { Gibbs potential, } \mathrm{J} \\ I & \text { interaction } \\ M & \text { moli or mass, } \mathrm{Kg}, \\ n & \text { constituent } \\ P & \text { pressure, Pa } \\ Q & \text { heat, } \mathrm{J} \\ R & \text { reservoir } \\ R & \text { universal gas constant } \\ S & \text { entropy, } \mathrm{J} /{ }^{\circ} \mathrm{K} \\ T & \text { temperature, }{ }^{\circ} \mathrm{K}\end{array}$




$\begin{array}{ll}U & \text { internal energy, } \mathrm{J} \\ V & \text { volume, cubic m } \\ W & \text { work, } \mathrm{J} \\ x & \text { mole }\end{array}$

\section{Greek symbols}
$\mu$
chemical potential, $\mathrm{J}$
$\Omega$
available energy, $\mathrm{J}$

\section{Subscripts and superscripts}

$\begin{array}{ll}A R & \text { composite system-reservoir } \\ C & \text { chemical } \\ G & \text { generalized } \\ M & \text { mechanical } \\ M A X & \text { maximum } \\ M I N & \text { minimum } \\ N E T & \text { net } \\ R & \text { reference system or reservoir } \\ R E V & \text { reversible } \\ T & \text { thermal } \\ 0 & \text { initial state } \\ 1 & \text { final state } \\ \rightarrow & \text { interaction flow outward } \\ \leftarrow & \text { interaction flow inward }\end{array}$

\section{Conflicts of Interest}

The author declares no conflicts of interest.

\section{References}

1. Gyftopoulos, E.; Beretta G.P. Thermodynamics: Foundations and Applications; Dover Publications: New York, NY, USA, 2005.

2. Gyftopoulos, E.P. Entropy: An Inherent, Non-statistical Property of any System in any State. Int. J. Thermodyn. 2006, 9, 107-115.

3. Beretta, G.P. Axiomatic Definition of Entropy for Nonequilibrium States. Int. J. Thermodyn. 2008, 11, 39-48.

4. Zanchini, E.; Barletta, A. Finite-mass heat reservoir and the second law. Il Nuovo Cimento 1995, 10, 1245-1258.

5. Zanchini, E.; Beretta, G.P. Removing Heat and Conceptual Loops from the Definition of Entropy. Int. J. Thermodyn. 2010, 13, 67-76. 
6. Smith, C.E.; von Spakovsky, M.R. Comparison of the non-equilibrium predictions of Intrinsic Quantum Thermodynamics at the atomistic level with experimental evidence. J. Phys. Conf. Ser. 2012, 380, 012015.

7. Pogliani, L.; Berberan-Santos, M.N. Constantin Carathéodori and the Axiomatic Thermodynamics. J. Mathemat. Chem. 2000, 28, 1-3.

8. Zanchini, E. Highest-entropy, Lowest-energy and Lowest-volume Principles. Int. J. Thermodyn. 2010, 39, 110-116.

9. Kotas, T.J. The Exergy Method of Thermal Plant Analysis; Krieger Publishing Company: Malabar, FL, USA, 1995.

10. Moran, M.J.; Sciubba, E. Exergy Analysis: Principles and Practice. J. Eng. Gas Turbines Power 1994, 116, 285-290.

11. Palazzo, P. Thermal and Mechanical Aspect of Entropy-Exergy Relationship. Int. J. Energy Environ. Eng. 2012, doi: 10.1186/2251-6832-3-4.

12. Zanchini, E.; Beretta, G.P. Recent Progress in the Definition of Thermodynamic Entropy. Entropy 2014, 16, 1547-1570.

13. Beretta, G.P.; Zanchini, E. A Definition of Thermodynamic Entropy Valid for Non-equilibrium States and Few-particle Systems. 2014, arXiv:1411.5395.

14. Palazzo, P. Theorem of Necessity and Sufficiency of Stable Equilibrium for Generalized Potential Equality between System and Reservoir. J. Mod. Phys. 2014, 5, 2003-2011.

15. Lucia, U. The Gouy-Stodola Theorem in Bioenergetic Analysis of Living Systems (Irreversibility in Bioenergetics of Living Systems). Energies 2014, 7, 5717-5739.

16. Sciubba, E. Entropy Generation Minima in Different Configurations of the Branching of a Fluid-Carrying Pipe in Laminar Isothermal Flow. Entropy 2010, 12, 1885-1866.

17. Sciubba, E. Entropy Generation Minimization as a Design Tool. Part 1: Analysis of Different Configurations of Branched and Non-branched Laminar Isothermal Flow through a Circular Pipe. Int. J. Thermodyn. 2011, 14, 11-20.

18. Sciacovelli, A.; Verda, V.; Sciubba, E. Entropy generation analysis as a design tool-A review. Renew. Sustain. Energy Rev. 2015, 43, 1167-1181.

19. Demirel, Y. Nonequilibrium thermodynamics modeling of coupled biochemical cycles in living cells. J. Non-Newton. Fluid Mech. 2010, 165, 953-972.

20. Demirel, Y. Nonequilibrium Thermodynamics. Transport and Rate Processes in Physica, Chemical and Biological Systems, 3rd ed.; Elsevier: Amsterdam, The Netherlands, 2014.

21. Demirel, Y. Exergy use in bioenergetics. Int. J. Exergy. 2004, 1, 128-146

22. Hatsopoulos, G.N.; Beretta, G.P. Where is the entropy challenge? In Proceedings of the International Thermodynamics Symposium in Honor and Memory of Professor Joseph H. Keenan, Cambridge, MA, USA, 4-5 October 2007; Beretta, G.P., Ghoniem, A.F., Hatsopoulos, G.N., Eds.; AIP CP Series, 2008; Volume 2033, pp. 34-54.

23. Baez, J.C.; Pollard, B.S. Quantropy. Entropy 2015, 17, 772-789.

(C) 2015 by the authors; licensee MDPI, Basel, Switzerland. This article is an open access article distributed under the terms and conditions of the Creative Commons Attribution license (http://creativecommons.org/licenses/by/4.0/). 